\title{
Curvature Properties of Quasi-Para-Sasakian Manifolds
}

\author{
I. Küpeli Erken
}

(Communicated by Uday Chand De)

\begin{abstract}
The paper is devoted to study quasi-para-Sasakian manifolds. Basic properties of such manifolds are obtained and general curvature identities are investigated. Next it is proved that if $M$ is a quasipara-Sasakian manifold of constant curvature $K$. Then $K \leq 0$ and $(i)$ if $K=0$, the manifold is paracosymplectic, (ii) if $K<0$, the quasi-para-Sasakian structure of $M$ is obtained by a homothetic deformation of a para-Sasakian structure. Finally, an example of a 3-dimensional proper quasipara-Sasakian manifold is constructed.
\end{abstract}

Keywords: quasi-para-Sasakian manifold; paracosymplectic manifold; constant curvature.

AMS Subject Classification (2010): Primary: 53B30; Secondary: 53D10; 53D15.

\section{Introduction}

Almost paracontact metric structures are the natural odd-dimensional analogue to almost paraHermitian structures, just like almost contact metric structures correspond to the almost Hermitian ones. The study of almost paracontact geometry was introduced by Kaneyuki and Williams in [8] and then it was continued by many other authors. A systematic study of almost paracontact metric manifolds was carried out in one of Zamkovoy's papers [17]. Comparing with the huge literature in almost contact geometry, it seems that there are necessities for new studies in almost paracontact geometry. Therefore, paracontact metric manifolds have been studied in recent years by many authors, emphasizing similarities and differences with respect to the most well known contact case. Interesting papers connecting these fields are, for example, [5], [4], [15], [17], and references therein.

Z. Olszak studied normal almost contact metric manifolds of dimension 3 [12]. He derived certain necessary and sufficient conditions for an almost contact metric structure on manifold to be normal and curvature properties of such structures and normal almost contact metric structures on a manifold of constant curvature were studied. Recently, J. Wełyczko studied curvature and torsion of Frenet-Legendre curves in 3-dimensional normal almost paracontact metric manifolds [16] and then normal almost paracontact metric manifolds were studied in [1], [9], [10].

The notion of quasi-Sasakian manifolds, introduced by D. E. Blair in [2], unifies Sasakian and cosymplectic manifolds. By definition, a quasi-Sasakian manifold is a normal almost contact metric manifold whose fundamental 2-form $\Phi:=g(\cdot, \phi \cdot)$ is closed. Quasi-Sasakian manifolds can be viewed as an odd-dimensional counterpart of Kaehler structures. These manifolds have been studied by several authors (e.g. [7], [11], [14]).

Although quasi-Sasakian manifolds were studied by several different authors and are considered a wellestablished topic in contact Riemannian geometry, to the author's knowledge, there do not exist any comprehensive study about quasi-para-Sasakian manifolds.

Motivated by these considerations, in this paper we make the first contribution to investigate basic properties and general curvature identities of quasi-para-Sasakian manifolds.

The paper is organized in the following way.

Section 2 is preliminary section, where we recall the definition of almost paracontact metric manifold and quasi-para-Sasakian manifolds.

In Section 3, we study basic properties and curvature identities of such manifolds. 
In the short auxiliary Section $4, D$-homothetic deformations of quasi-para-Sasakian structures are studied and in Section 5, we characterize quasi-para-Sasakian manifolds of constant curvature. Finally, an example of 3 -dimensional proper quasi-para-Sasakian manifold is given.

\section{Preliminaries}

Let $M$ be a $(2 n+1)$-dimensional differentiable manifold and $\phi$ is a $(1,1)$ tensor field, $\xi$ is a vector field and $\eta$ is a one-form on $M$. Then $(\phi, \xi, \eta)$ is called an almost paracontact structure on $M$ if

(i) $\phi^{2}=I d-\eta \otimes \xi, \quad \eta(\xi)=1$,

(ii) the tensor field $\phi$ induces an almost paracomplex structure on the distribution $D=$ ker $\eta$, that is the eigendistributions $D^{ \pm}$, corresponding to the eigenvalues \pm 1 , have equal dimensions, $\operatorname{dim} D^{+}=$ $\operatorname{dim} D^{-}=n$.

The manifold $M$ is said to be an almost paracontact manifold if it is endowed with an almost paracontact structure [17].

Let $M$ be an almost paracontact manifold. $M$ will be called an almost paracontact metric manifold if it is additionally endowed with a pseudo-Riemannian metric $g$ of a signature $(n+1, n)$, i.e.

$$
g(\phi X, \phi Y)=-g(X, Y)+\eta(X) \eta(Y) .
$$

For such manifold, we have

$$
\eta(X)=g(X, \xi), \phi(\xi)=0, \eta \circ \phi=0 .
$$

Moreover, we can define a skew-symmetric tensor field (a 2 -form) $\Phi$ by

$$
\Phi(X, Y)=g(X, \phi Y)
$$

usually called fundamental form.

For an almost paracontact manifold, there exists an orthogonal basis $\left\{X_{1}, \ldots, X_{n}, Y_{1}, \ldots, Y_{n}, \xi\right\}$ such that $g\left(X_{i}, X_{j}\right)=\delta_{i j}, g\left(Y_{i}, Y_{j}\right)=-\delta_{i j}$ and $Y_{i}=\phi X_{i}$, for any $i, j \in\{1, \ldots, n\}$. Such basis is called a $\phi$-basis.

On an almost paracontact manifold, one defines the $(1,2)$-tensor field $N^{(1)}$ by

$$
N^{(1)}(X, Y)=[\phi, \phi](X, Y)-2 d \eta(X, Y) \xi,
$$

where $[\phi, \phi]$ is the Nijenhuis torsion of $\phi$

$$
[\phi, \phi](X, Y)=\phi^{2}[X, Y]+[\phi X, \phi Y]-\phi[\phi X, Y]-\phi[X, \phi Y] .
$$

If $N^{(1)}$ vanishes identically, then the almost paracontact manifold (structure) is said to be normal [17]. The normality condition says that the almost paracomplex structure $J$ defined on $M \times \mathbb{R}$

$$
J\left(X, \lambda \frac{d}{d t}\right)=\left(\phi X+\lambda \xi, \eta(X) \frac{d}{d t}\right)
$$

is integrable.

If $d \eta(X, Y)=g(X, \phi Y)=\Phi(X, Y)$, then $(M, \phi, \xi, \eta, g)$ is said to be paracontact metric manifold. In a paracontact metric manifold one defines a symmetric, trace-free operator $h=\frac{1}{2} \mathcal{L}_{\xi} \phi$, where $\mathcal{L}_{\xi}$, denotes the Lie derivative. It is known [17] that $h$ anti-commutes with $\phi$ and satisfies $h \xi=0, \operatorname{tr} h=\operatorname{tr} h \phi=0$ and $\nabla \xi=-\phi+\phi h$, where $\nabla$ is the Levi-Civita connection of the pseudo-Riemannian manifold $(M, g)$.

Moreover $h=0$ if and only if $\xi$ is Killing vector field. In this case $(M, \phi, \xi, \eta, g)$ is said to be a K-paracontact manifold. Similarly as in the class of almost contact metric manifolds [3], a normal almost paracontact metric manifold will be called para-Sasakian if $\Phi=d \eta[6]$. Also in this context the para-Sasakian condition implies the $K$-paracontact condition and the converse holds only in dimension 3.

Definition 2.1. An almost paracontact metric manifold $\left(M^{2 n+1}, \phi, \xi, \eta, g\right)$ is called quasi-para-Sasakian if the structure is normal and its fundamental 2 -form $\Phi$ is closed.

The class of para-Sasakian manifolds is contained in the class of quasi-para-Sasakian manifolds. The converse does not hold in general. A paracontact metric manifold will be called paracosymplectic if $d \Phi=0, d \eta=0$ [5], also, the class of paracosymplectic manifolds is contained in the class of quasi-para-Sasakian manifolds. 


\section{Basic Structure and Curvature Identities}

Definition 3.1. For a quasi-para-Sasakian manifold $\left(M^{2 n+1}, \phi, \xi, \eta, g\right)$, define the $(1,1)$ tensor field $\mathcal{A}$ by

$$
\mathcal{A} X=\nabla_{X} \xi \text {. }
$$

Remark 3.1. For the easy readability of the identities, we will use $g(\mathcal{A} X, Y)=\left(\nabla_{X} \eta\right) Y$.

Since the proof of the following Lemma is quite similar to Lemma 4.1 of [2], we don't give the proof of it.

Lemma 3.1. Vector field $\xi$ of a quasi-para-Sasakian structure $(\phi, \xi, \eta, g)$ is a Killing vector field.

$$
g(\mathcal{A} X, Y)+g(X, \mathcal{A} Y)=0 .
$$

Proposition 3.1. For a quasi-para-Sasakian manifold $\left(M^{2 n+1}, \phi, \xi, \eta, g\right)$, we have

$$
\begin{gathered}
\left(\nabla_{X} \phi\right) Y=-g(\mathcal{A} X, \phi Y) \xi-\eta(Y) \phi \mathcal{A} X, \\
\nabla_{\xi} \phi=0, \quad \nabla_{\xi} \xi=0, \quad \nabla_{\xi} \eta=0, \\
\mathcal{A} \phi X=\phi \mathcal{A} X, \\
g(\mathcal{A} \phi X, \phi Y)=-g(\mathcal{A} X, Y), \\
g(\mathcal{A} \phi X, Y)=-g(\mathcal{A} X, \phi Y),
\end{gathered}
$$

where $X, Y$ are arbitrary vector fields on $M^{2 n+1}$.

Proof. Using the Cartan magic formula

$$
\mathcal{L}_{\xi} \Phi=d\left(i_{\xi} \Phi\right)+i_{\xi}(d \Phi)
$$

we find $\mathcal{L}_{\xi} \Phi=0$, since $d \Phi=0$ and $\left(i_{\xi} \Phi\right) X=\Phi(\xi, X)=g(\xi, \phi X)=0$, where $\mathcal{L}$ indicates the operator of the Lie differentiation. If we use the definition of quasi-para-Sasakian manifold, (3.2) and the well known equation $2 d \eta(X, Y)=X(\eta(Y))-Y(\eta(X))-\eta([X, Y])$ in Proposition 2.4 of [17], we obtain (3.3).

$\mathcal{L}_{\xi} \Phi=0$, properties of $\phi$ and (3.4) follow

$$
\begin{aligned}
\left(\mathcal{L}_{\xi} \Phi\right)(X, Y) & =\mathcal{L}_{\xi} \Phi(X, Y)-\Phi\left(\mathcal{L}_{\xi} X, Y\right)-\Phi\left(X, \mathcal{L}_{\xi} Y\right) \\
0 & =g(\phi \mathcal{A} Y-\mathcal{A} \phi Y, X) .
\end{aligned}
$$

So we obtain (3.5). In virtue of (3.5), we obtain (3.6) and (3.7).

Lemma 3.2. For a quasi-para-Sasakian manifold $\left(M^{2 n+1}, \phi, \xi, \eta, g\right)$ with its curvature transformation $R_{X Y}=$ $\left[\nabla_{X}, \nabla_{Y}\right]-\nabla_{[X, Y]}$, the following equations hold

$$
\begin{gathered}
R(\xi, X) Y=-\left(\nabla_{X} \mathcal{A}\right) Y, \\
g(R(\xi, X) Y, \xi)=g(\mathcal{A} X, \mathcal{A} Y), \\
g(R(\xi, X) \phi Y, \phi Z)+g(R(\xi, X) Y, Z)=g(\mathcal{A} X, \mathcal{A} Y) \eta(Z)-g(\mathcal{A} X, \mathcal{A} Z) \eta(Y), \\
S(\xi, \xi)=-t r \mathcal{A}^{2} .
\end{gathered}
$$

Proof. Using the fact that $\xi$ is Killing vector field and (3.2), one can easily get (3.8). If we take the inner product of (3.8) with $\xi$ and then use (3.2), we have (3.9). Using (3.3), we get

$$
\begin{aligned}
\mathcal{A} \nabla_{X} \phi Y & =-\eta(Y) \phi \mathcal{A}^{2} X+\phi \mathcal{A} \nabla_{X} Y, \\
\mathcal{A}\left(\nabla_{X} \phi\right) Y & =-\eta(Y) \phi \mathcal{A}^{2} X .
\end{aligned}
$$

From (3.5) and (3.3), we have

$$
\phi \nabla_{X} \mathcal{A} Y-\nabla_{X} \mathcal{A} \phi Y=-\left(\nabla_{X} \phi\right) \mathcal{A} Y=g(\mathcal{A} X, \phi \mathcal{A} Y) \xi .
$$

Taking into account (3.8), (3.12) and (3.13), we obtain

$$
R(\xi, X) \phi Y-\phi R(\xi, X) Y=-\eta(Y) \phi \mathcal{A}^{2} X+g(\mathcal{A} X, \phi \mathcal{A} Y) \xi .
$$

On the other hand, if we take the inner product of (3.14) with $\phi Z$ and use (3.2) and (3.9), we get (3.10). The proof of (3.11) is a direct consequence of (3.9). 
Proposition 3.2. For a quasi-para-Sasakian manifold $\left(M^{2 n+1}, \phi, \xi, \eta, g\right)$, we also have

$$
\begin{aligned}
g(R(X, Y) \phi Z, \phi W)+g(R(X, Y) Z, W)= & \eta(W) g(R(X, Y) Z, \xi)+\eta(Z) g(R(X, Y) \xi, W) \\
& -g(\mathcal{A} X, \phi W) g(\mathcal{A} Y, \phi Z)+g(\mathcal{A} X, \phi Z) g(\mathcal{A} Y, \phi W) \\
& +g(\mathcal{A} X, Z) g(\mathcal{A} Y, W)-g(\mathcal{A} X, W) g(\mathcal{A} Y, Z) .
\end{aligned}
$$

Proof. The following formula is valid

$$
\left(\nabla_{X} \nabla_{Y} \phi\right) Z=\nabla_{X}\left(\nabla_{Y} \phi\right) Z-\left(\nabla_{\nabla_{X}} \phi\right) Z-\left(\nabla_{Y} \phi\right) \nabla_{X} Z
$$

Now we suppose that $P$ is a fixed point of $\left(M^{2 n+1}, \phi, \xi, \eta, g\right)$ and $X, Y, Z$ are vector fields such that $(\nabla X)_{P}=$ $(\nabla Y)_{P}=(\nabla Z)_{P}=0$. Hence the last identity at the point $P$, reduces to the form

$$
\left(\nabla_{X} \nabla_{Y} \phi\right) Z=\nabla_{X}\left(\nabla_{Y} \phi\right) Z-\left(\nabla_{Y} \phi\right) \nabla_{X} Z
$$

Now, after differentiating (3.3) covariantly and using (3.16), we find

$$
\left(\nabla_{X} \nabla_{Y} \phi\right) Z=-g\left(\nabla_{X} \mathcal{A} Y, \phi Z\right) \xi-g(\mathcal{A} Y, \phi Z) \mathcal{A} X-g(\mathcal{A} X, Z) \phi \mathcal{A} Y-\eta(Z) \phi \nabla_{X} \mathcal{A} Y \text {. }
$$

On the other hand, combining the last equation and (3.3), we obtain

$$
\begin{aligned}
(R(X, Y) \phi) Z= & \left(\nabla_{X} \nabla_{Y} \phi\right) Z-\left(\nabla_{Y} \nabla_{X} \phi\right) Z-\left(\nabla_{[X, Y]} \phi\right) Z \\
= & -g(R(X, Y) \xi, \phi Z) \xi-\eta(Z) \phi R(X, Y) \xi \\
& -g(\mathcal{A} Y, \phi Z) \mathcal{A} X+g(\mathcal{A} X, \phi Z) \mathcal{A} Y \\
& -g(\mathcal{A} X, Z) \phi \mathcal{A} Y+g(\mathcal{A} Y, Z) \phi \mathcal{A} X .
\end{aligned}
$$

Taking into account (2.1), we deduce

$$
g(R(X, Y) \phi Z, \phi W)+g(R(X, Y) Z, W)=g(R(X, Y) Z, \xi) \eta(W)+g((R(X, Y) \phi) Z, \phi W) .
$$

Taking the inner product of (3.17) with $\phi W$, and using the above equation, we get (3.15).

Proposition 3.3. A quasi-para-Sasakian manifold $\left(M^{2 n+1}, \phi, \xi, \eta, g\right)$ satisfies followings

$$
\begin{aligned}
S^{*}(Y, Z)+S(Y, Z)= & S(Y, \xi) \eta(Z)+g(\mathcal{A} Y, \phi Z) \operatorname{trace}(\phi \mathcal{A}) \\
& -g(\mathcal{A} Y, \mathcal{A} Z) \\
r^{*}+r= & -\operatorname{tr}^{2}(\phi \mathcal{A})
\end{aligned}
$$

where $S^{*}(X, Y)=\sum_{i=1}^{2 n+1} \varepsilon_{i} g\left(R\left(e_{i}, X\right) \phi Y, \phi e_{i}\right)$ denotes the ${ }^{*}$-Ricci curvature tensor and $r^{*}=\sum_{i=1}^{2 n+1} \varepsilon_{i} S^{*}\left(e_{i}, e_{i}\right)$ denotes the *-scalar curvature of the $(M, \phi, \xi, \eta, g)$, where $\left\{e_{i}\right\}, i \in\{1, \ldots, 2 n+1\}$ be a local $\phi$-basis.

Proof. One can show that $\sum_{i=1}^{2 n+1} g\left(\mathcal{A} e_{i}, e_{i}\right)=0$. Using (3.2), (3.5) and (3.7), we get

$$
\begin{aligned}
\sum_{i=1}^{2 n+1} g\left(\mathcal{A} e_{i}, \phi Z\right) g\left(\mathcal{A} Y, \phi e_{i}\right) & =\sum_{i=1}^{2 n+1} g\left(\mathcal{A} \phi Y, e_{i}\right) g\left(\mathcal{A} \phi Z, e_{i}\right) \\
& =g(\mathcal{A} \phi Y, \mathcal{A} \phi Z)=-g(\mathcal{A} Y, \mathcal{A} Z) .
\end{aligned}
$$

Using the fact that $\operatorname{tr}(\phi \mathcal{A})=\sum_{i=1}^{2 n+1} \varepsilon_{i} g\left(\phi \mathcal{A} e_{i}, e_{i}\right)=-\sum_{i=1}^{2 n+1} g\left(\mathcal{A} e_{i}, \phi e_{i}\right),(3.9)$ and (3.20) after replacing $X, W$ by $e_{i}$ in (3.15) and taking summation over $i$, we find (3.18). For the proof of (3.19), after replacing $Y, Z$ by $e_{i}$ in (3.18) and taking the summation over $i$, and using (3.11), we obtain the requested equation. 


\section{D-homothetic deformations}

Let $\left(M^{2 n+1}, \phi, \xi, \eta, g\right)$ be an almost paracontact metric manifold and $(\phi, \xi, \eta, g)$ is an almost paracontact metric structure on $\left(M^{2 n+1}, \phi, \xi, \eta, g\right)$. Tensor fields $\tilde{\phi}, \tilde{\xi}, \tilde{\eta}$ and $\tilde{g}$ defined as

$$
\tilde{\phi}=\phi, \quad \tilde{\xi}=\frac{1}{\alpha} \xi, \tilde{\eta}=\alpha \eta, \quad \tilde{g}=\beta g+\left(\alpha^{2}-\beta\right) \eta \otimes \eta,
$$

where $\alpha \neq 0$ and $\beta>0$.

Thus, $(\tilde{\phi}, \tilde{\xi}, \tilde{\eta}, \tilde{g})$ is also an almost paracontact metric structure on $\left(M^{2 n+1}, \phi, \xi, \eta, g\right)$.

If the almost paracontact metric structures $(\phi, \xi, \eta, g)$ and $(\tilde{\phi}, \tilde{\xi}, \tilde{\eta}, \tilde{g})$ are related with $(4.1)$, then $(\tilde{\phi}, \tilde{\xi}, \tilde{\eta}, \tilde{g})$ is said to be $D$-homothetic to $(\phi, \xi, \eta, g)$, namely, the almost paracontact metric structure $(\tilde{\phi}, \tilde{\xi}, \tilde{\eta}, \tilde{g})$ is obtained by a $D$-homothetic deformation of the almost paracontact metric structure $(\phi, \xi, \eta, g)$. If $\alpha^{2}=\beta$, then $D$-homothetic deformation will be called homothetic deformation [13].

Proposition 4.1. If $(\phi, \xi, \eta, g)$ is a quasi-para-Sasakian structure, then the structure $(\tilde{\phi}, \tilde{\xi}, \tilde{\eta}, \tilde{g})$ is also quasi-paraSasakian. If $(\phi, \xi, \eta, g)$ is para-Sasakian, then $(\tilde{\phi}, \tilde{\xi}, \tilde{\eta}, \tilde{g})$ is para-Sasakian if and only if $\alpha=\beta$.

Proof. By virtue of Definition 2.1 and (4.1), we obtain the assertion.

Lemma 4.1. Let $(\tilde{\phi}, \tilde{\xi}, \tilde{\eta}, \tilde{g})$ be a quasi-para-Sasakian structure obtained from $(\phi, \xi, \eta, g)$ by a D-homothetic deformation. Then we have the following relation between the Levi-Civita connections $\tilde{\nabla}$ and $\nabla$ with respect to $\tilde{g}$ and $g$.

$$
\tilde{\nabla}_{X} Y=\nabla_{X} Y+\left(\frac{\alpha^{2}}{\beta}-1\right)(\eta(Y) \mathcal{A} X+\eta(X) \mathcal{A} Y) .
$$

Proof. By Koszul formula we have

$$
\begin{aligned}
2 \tilde{g}\left(\tilde{\nabla}_{X} Y, Z\right)= & X \tilde{g}(Y, Z)+Y \tilde{g}(X, Z)-Z \tilde{g}(X, Y) \\
& +\tilde{g}([X, Y], Z)+\tilde{g}([Z, X], Y)+\tilde{g}([Z, Y], X),
\end{aligned}
$$

for any vector fields $X, Y, Z$. Using $\tilde{g}=\beta g+\left(\alpha^{2}-\beta\right) \eta \otimes \eta$ and (3.2) in the last equation, we obtain

$$
2 \tilde{g}\left(\tilde{\nabla}_{X} Y, Z\right)=2 \beta g\left(\nabla_{X} Y, Z\right)+2\left(\alpha^{2}-\beta\right)\left[\eta\left(\nabla_{X} Y\right) \eta(Z)+g(Z, \mathcal{A} X) \eta(Y)+g(Z, \mathcal{A} Y) \eta(X)\right] .
$$

Since $g(Z, \mathcal{A} Y)=g(\mathcal{A} Y, Z)$, we get

$$
\begin{aligned}
2 \tilde{g}\left(\tilde{\nabla}_{X} Y, Z\right)= & 2 \beta g\left(\nabla_{X} Y, Z\right) \\
& +2\left(\alpha^{2}-\beta\right)\left[\eta(X) g(\mathcal{A} Y, Z)+\eta(Y) g(\mathcal{A} X, Z)+\eta(Z) \eta\left(\nabla_{X} Y\right)\right] .
\end{aligned}
$$

Moreover, $\tilde{g}\left(\tilde{\nabla}_{X} Y, Z\right)$ is equal to

$$
\beta g\left(\tilde{\nabla}_{X} Y, Z\right)+\left(\alpha^{2}-\beta\right) \eta\left(\tilde{\nabla}_{X} Y\right) \eta(Z) .
$$

Substituting (4.4) in (4.3), we obtain

$$
\begin{aligned}
& \beta g\left(\tilde{\nabla}_{X} Y, Z\right)+\left(\alpha^{2}-\beta\right) \eta\left(\tilde{\nabla}_{X} Y\right) \eta(Z) \\
= & \beta g\left(\nabla_{X} Y, Z\right)+\left(\alpha^{2}-\beta\right)\left[\eta(X) g(\mathcal{A} Y, Z)+\eta(Y) g(\mathcal{A} X, Z)+\eta(Z) \eta\left(\nabla_{X} Y\right)\right] .
\end{aligned}
$$

Setting $Z=\xi$ in (4.5) and using (3.2), we get

$$
\eta\left(\tilde{\nabla}_{X} Y\right)=\eta\left(\nabla_{X} Y\right)
$$

(4.2) is a direct consequence of (4.5) and (4.6).

Proposition 4.2. Let $\left(M^{2 n+1}, \phi, \xi, \eta, g\right)$ and $\left(\tilde{M}^{2 n+1}, \tilde{\phi}, \tilde{\xi}, \tilde{\eta}, \tilde{g}\right)$ are locally D-homothetic quasi-para-Sasakian manifolds. Then following identities hold:

$$
\begin{aligned}
\tilde{\mathcal{A}} X & =\frac{\alpha}{\beta} \mathcal{A} X, \\
\tilde{g}(\mathcal{A} X, Y) & =\alpha g(\mathcal{A} X, Y),
\end{aligned}
$$




$$
\begin{aligned}
\tilde{R}(X, Y) Z= & R(X, Y) Z \\
& -\left(\frac{\alpha^{2}}{\beta}-1\right)\{g(\mathcal{A} Y, Z) \mathcal{A} X-g(\mathcal{A} X, Z) \mathcal{A} Y-2 g(\mathcal{A} X, Y) \mathcal{A} Z\} \\
& +\left(\frac{\alpha^{2}}{\beta}-1\right)^{2}\left\{\eta(X) \eta(Z) \mathcal{A}^{2} Y-\eta(Y) \eta(Z) \mathcal{A}^{2} X\right\} \\
& +\left(\frac{\alpha^{2}}{\beta}-1\right)\{\eta(X) R(\xi, Y) Z+\eta(Y) R(X, \xi) Z+\eta(Z) R(X, Y) \xi\},
\end{aligned}
$$

for any vector fields $X, Y, Z$.

Proof. After setting $Y=\xi$ in (4.2), if we use $\tilde{\xi}=\frac{1}{\alpha} \xi$ and (3.4), we get (4.7). Using (4.1) and (4.2), after some calculations one can obtain (4.8). From the curvature formula

$$
\tilde{R}(X, Y) Z=\left[\tilde{\nabla}_{X}, \tilde{\nabla}_{Y}\right] Z-\tilde{\nabla}_{[X, Y]} Z
$$

Eq. (3.2), (3.8) and (4.2), after a straightforward computation one can get (4.9).

Since the proof of the following proposition is quite similar to Proposition 4.4 of [11], we don't give the proof of it.

Proposition 4.3. Let $(\phi, \xi, \eta, g)$ be a quasi-para-Sasakian structure. Then the following assertions are equivalent to each other:

i) $(\phi, \xi, \eta, g)$ can be obtained by a D-homothetic deformation of a para-Sasakian structure,

ii) $(\phi, \xi, \eta, g)$ can be obtained by a homothetic deformation of a para-Sasakian structure,

iii) $\mathcal{A} X=\lambda \phi X$, for $\lambda=$ const. $\neq 0$.

\section{Quasi-Para-Sasakian manifolds of constant curvature}

Theorem 5.1. Let $\left(M^{2 n+1}, \phi, \xi, \eta, g\right)$ be a quasi-para-Sasakian manifold of constant curvature $K$. Then $K \leq 0$. Furthermore,

-If $K=0$, the manifold is paracosymplectic,

-If $K<0$, the structure $(\phi, \xi, \eta, g)$ is obtained by a homothetic deformation of a para-Sasakian structure on $M^{2 n+1}$.

Proof. One can see that $K \leq 0$ from Lemma 3.2. If $K=0$, by (3.11), we get $\mathcal{A}=0$. Hence, from (3.3), we have $\nabla \phi=0$. This means the manifold is paracosymplectic. Assume that $K<0$. The claim follows from Proposition 4.3. So, we should obtain $\mathcal{A} X=\lambda \phi X$, for $\alpha=$ const. $\neq 0$. After straightforward calculations, we have $r=2 n(2 n+1) K$ and $r^{*}=-2 n K$. If we use these equations in (3.19), we obtain

$$
\operatorname{tr}(\phi \mathcal{A})=2 n \lambda, \text { so } K=-\lambda^{2} .
$$

By direct calculations, we get

$$
\begin{aligned}
S(Y, Z) & =2 n K g(Y, Z) \text { and } \\
S^{*}(Y, Z) & =K(-g(Y, Z)+\eta(Y) \eta(Z)) \stackrel{(3.9)}{=}-g(\mathcal{A} Y, \mathcal{A} Z) .
\end{aligned}
$$

Making use of (5.1) and (5.2) in (3.18), we deduce

$$
g(\mathcal{A} Y, \phi Z)=-\lambda(g(Y, Z)-\eta(Y) \eta(Z)) .
$$

Putting $\phi Y$ for $Y$ in (5.3) and using (3.6), we have $\mathcal{A} Y=\lambda \phi Y$. This completes the proof.

\section{Example}

Now, we will give an example of 3-dimensional proper quasi-para-Sasakian manifold. 
Example 6.1. We consider the 3-dimensional manifold

$$
M^{3}=\left\{(x, y, z) \in \mathbb{R}^{3}, z \neq 0\right\}
$$

and the vector fields

$$
\phi e_{2}=e_{1}=-4 y \frac{\partial}{\partial x}+z \frac{\partial}{\partial z}, \quad \phi e_{1}=e_{2}=\frac{\partial}{\partial y}, \quad \xi=e_{3}=\frac{\partial}{\partial x} .
$$

The 1-form $\eta=d x+\frac{4 y}{z} d z$ defines an almost paracontact structure on $M$ with characteristic vector field $\xi=\frac{\partial}{\partial x}$. Let $g$, $\phi$ be the semi-Riemannian metric $\left(g\left(e_{1}, e_{1}\right)=-g\left(e_{2}, e_{2}\right)=g(\xi, \xi)=1\right)$ and the $(1,1)$-tensor field respectively given by

$$
\begin{aligned}
g & =\left(\begin{array}{ccc}
1 & 0 & \frac{2 y}{z} \\
0 & -1 & 0 \\
\frac{2 y}{z} & 0 & \frac{1+28 y^{2}}{z^{2}}
\end{array}\right), \\
\phi & =\left(\begin{array}{ccc}
0 & -4 y & 0 \\
0 & 0 & \frac{1}{z} \\
0 & z & 0
\end{array}\right),
\end{aligned}
$$

with respect to the basis $\frac{\partial}{\partial x}, \frac{\partial}{\partial y}, \frac{\partial}{\partial z}$.

Using $\nabla_{X} \xi=\beta \phi X($ see $[16])$ we have

$$
\begin{array}{ccc}
\nabla_{e_{1}} e_{1}=0, & \nabla_{e_{2}} e_{1}=-2 \xi, & \nabla_{\xi} e_{1}=2 e_{2} \\
\nabla_{e_{1}} e_{2}=2 \xi, & \nabla_{e_{2}} e_{2}=0, & \nabla_{\xi} e_{2}=2 e_{1} \\
\nabla_{e_{1}} \xi=2 e_{2}, & \nabla_{e_{2}} \xi=2 e_{1}, & \nabla_{\xi} \xi=0
\end{array}
$$

Hence the manifold is a 3-dimensional quasi-para-Sasakian manifold with $\beta=2$. Using the above equations, we obtain

$$
\begin{array}{ccc}
R\left(e_{1}, e_{2}\right) \xi=0, & R\left(e_{2}, \xi\right) \xi=-4 e_{2}, & R\left(e_{1}, \xi\right) \xi=-4 e_{1}, \\
R\left(e_{1}, e_{2}\right) e_{2}=-12 e_{1}, & R\left(e_{2}, \xi\right) e_{2}=-4 \xi, & R\left(e_{1}, \xi\right) e_{2}=0, \\
R\left(e_{1}, e_{2}\right) e_{1}=-12 e_{2}, & R\left(e_{2}, \xi\right) e_{1}=0, & R\left(e_{1}, \xi\right) e_{1}=4 \xi .
\end{array}
$$

Using (6.1), we have constant scalar curvature as follows, $r=S\left(e_{1}, e_{1}\right)-S\left(e_{2}, e_{2}\right)+S(\xi, \xi)=8$. We want to remark that this example is neither the paracosymplectic manifold nor the para-Sasakian manifold example.

\section{References}

[1] Bejan, C. L. and Crasmareanu, M., Second order parallel tensors and Ricci solitons in 3-dimensional normal paracontact geometry. Ann. Global Anal. Geom. 46(2) (2014), 117-127.

[2] Blair, D. E., The theory of quasi-Sasakian structures. J. Differential Geom. 1 (1967), 331-345.

[3] Blair, D. E., Riemannian Geometry of Contact and Symplectic Manifolds. Progress in Mathematics Vol. 203, Birkhäuser. Boston 2002.

[4] Cappelletti-Montano, B., Küpeli Erken, I. and Murathan, C., Nullity conditions in paracontact geometry. Diff. Geom. Appl. 30 (2012), 665-693.

[5] Dacko, P., On almost para-cosymplectic manifolds. Tsukuba J. Math. 28 (2004), 193-213.

[6] Erdem, S., On almost (para)contact (hyperbolic) metric manifolds and harmonicity of $\left(\varphi, \varphi^{\prime}\right)$-holomorphic maps between them. Houston J. Math. 28 (2002), 21-45.

[7] Kanemaki, S., Quasi-Sasakian manifolds. Tohoku Math. J. 29 (1977), 227-233.

[8] Kaneyuki, S. and Williams, F. L., Almost paracontact and parahodge structures on manifolds. Nagoya Math. J. 99 (1985), $173-187$.

[9] Küpeli Erken, I., Some classes of 3-dimensional normal almost paracontact metric manifolds. Honam Math. J. 37(4) (2015), 457-468.

[10] Küpeli Erken, I., On normal almost paracontact metric manifolds of dimension 3. Facta Univ. Ser. Math. Inform. 30(5) (2015), 777-788.

[11] Olszak, Z., Curvature properties of quasi-Sasakian manifolds. Tensor. 38 (1982), 19-28.

[12] Olszak, Z., Normal almost contact metric manifolds of dimension three. Ann. Polon. Math. XLVII (1986), 41-50.

[13] Tanno, S., The topology of contact Riemannian manifolds. Illinois J. Math. 12 (1968), 700-717.

[14] Tanno, S., Quasi-Sasakian structures of rank 2p + 1. J. Differential Geom. 5 (1971), 317-324.

[15] Wełyczko, J., On basic curvature identities for almost (para)contact metric manifolds. Available in Arxiv: 1209.4731 [math. DG].

[16] Welyczko, J., On Legendre Curves in 3-Dimensional Normal Almost Paracontact Metric Manifolds. Result. Math. 54 (2009), $377-387$.

[17] Zamkovoy, S., Canonical connections on paracontact manifolds. Ann. Glob. Anal. Geom. 36 (2009), 37-60. 


\section{Affiliations}

I. KÜPELI ERKEN

ADDRESS: Bursa Technical University, Faculty of Engineering and Natural Sciences, Department of Mathematics, 16330, Bursa-Turkey.

E-MAIL: irem.erken@btu.edu.tr

ORCID ID: 0000-0003-4471-3291 